\title{
Sonolência diurna excessiva em adolescentes: prevalência e fatores associados
}

\author{
Daytime sleepiness in adolescents: prevalence and associated factors
}

\author{
Érico Felden Pereira ${ }^{1}$, Clarissa Stefani Teixeira² ${ }^{2}$ Fernando Mazzilli Louzada ${ }^{3}$
}

\section{RESUMO}

Objetivo: Analisar pesquisas sobre sonolência diurna excessiva em adolescentes e apresentar, por meio de revisão sistemática, as prevalências e os fatores associados.

Fontes de dados: Foram analisados estudos encontrados nas bases de dados PubMed e SciELO, que apresentassem resultados originais, sem restrições de período e idioma na pesquisa.

Síntese dos dados: A prevalência de sonolência diurna excessiva encontrada variou entre 7,8 e 55,8\%. As escalas Epworth Sleepiness Scale e a School Sleep Habits Survey foram os principais instrumentos utilizados. Associações da sonolência diurna excessiva com problemas relacionados ao sono, como o ranger de dentes, apneia e insônia, dentre outros, foram descritas com maior frequência nos estudos analisados. A associação de outros possíveis fatores de cunho biológico, ambiental e comportamental com a sonolência diurna excessiva não foi descrita com clareza.

Conclusões: Vários fatores, como o comportamento sedentário e hábitos alimentares, foram pouco investigados e apresentaram resultados divergentes quando analisados como variáveis associadas à sonolência diurna excessiva. Uma carência de investigações epidemiológicas com amostras brasileiras foi identificada.

Palavras-chave: transtornos do sono do ritmo circadiano; adolescente; epidemiologia.

\section{ABSTRACT}

Objective: To assess studies on excessive daytime sleepiness in adolescents and to present a systematic review the prevalence and associated factors.

Data sources: Papers found on PubMed and SciELO databases presenting original results without restriction of time and language were evaluated.

Data synthesis: The prevalence of excessive daytime sleepiness ranged from 7.8 to $55.8 \%$. The Epworth Sleepiness Scale and the School Sleep Habits Survey were the main tools used in the studies. Associations of excessive daytime sleepiness with sleeping-related problems, such as teeth gnashing, sleep apnea and insomnia, among others, were frequently described. The association of other biological, environmental and behavioral factors with excessive daytime sleepiness was not clearly assessed.

Conclusions: Many factors, such as sedentary behavior and alimentary habits, were poorly investigated so far and presented controversial results when analyzed as variables related to excessive daytime sleepiness. A lack of epidemiological studies with Brazilian adolescents was identified.

Key-words: sleep disorders, circadian rhythm; adolescent; epidemiology.
Instituição: Laboratório de Cronobiologia Humana da Universidade Federal do Paraná (UFPR), Curitiba, PR, Brasil

'Doutorando em Educação Física pela UFPR, Curitiba, PR, Brasil

2Doutoranda em Engenharia de Produção pela Universidade Federal de Santa Catarina (UFSC), Florianópolis, SC, Brasil

${ }^{3}$ Doutor; Professor do Departamento de Fisiologia da UFPR, Curitiba, PR, Brasil

Endereço para correspondência:

Érico Felden Pereira

Rua Otacílio Chaves, 253

CEP 97045-360 - Santa Maria/RS

E-mail: ericofelden@gmail.com
Fonte financiadora do projeto: Érico Felden Pereira é bolsista de doutorado da Coordenação de Aperfeiçoamento de Pessoal de Nível Superior (Capes) e Clarissa Stefani Teixeira é bolsista de doutorado do Conselho Nacional de Desenvolvimento Científico e Tecnológico (CNPq).

Conflito de interesse: nada a declarar

Recebido em: 17/3/09

Aprovado em: 4/7/09 


\section{Introdução}

Na última década e, principalmente, nos últimos anos, um maior número de estudos com amostras representativas de adolescentes com foco na sonolência diurna excessiva (SDE) e seus fatores associados têm sido publicados, embora uma carência de estudos epidemiológicos nesse contexto possa ser verificada $^{(1-3)}$. Não obstante, profissionais das áreas de saúde e educação, mesmo que de forma tímida, vêm se conscientizando da importância dos aspectos relacionados ao sono e sonolência para o aprendizado e saúde dos escolares ${ }^{(4)}$.

Divergências quanto à prevalência de sonolência diurna excessiva em adolescentes em função dos instrumentos de análise utilizados, populações investigadas e dos procedimentos adotados nas pesquisas são apontadas pela literatura ${ }^{(2,5-7)}$. A sonolência diurna excessiva é considerada uma sensação subjetiva de necessidade de sono e está associada à diminuição do rendimento no trabalho e na escola, afetando negativamente a aprendizagem, a interação social e a qualidade de $\operatorname{vida}^{(7,8)}$. É observada especialmente na adolescência, fase de vida marcada por mudanças biológicas, psíquicas e sociais importantes, na qual ocorre um atraso de fase mais evidente, ou seja, um atraso na expressão do ciclo vigília-sono marcado por horários de dormir mais tardios, o que pode dificultar a adaptação aos horários sociais, principalmente no final da puberdade $^{(9,10)}$.

O aumento da propensão à sonolência diurna excessiva na adolescência se deve a fatores biológicos, ambientais e comportamentais $^{(11)}$. As mudanças nos padrões de sono na adolescência estão associadas a um aumento das obrigações escolares e atividades sociais, além de atividades como televisão e internet, que podem colaborar para que os adolescentes deitem mais tarde e fiquem mais sonolentos ${ }^{(12)}$. Apesar disso, independentemente das mudanças nos horários do sono, a sonolência diurna excessiva apresenta uma tendência de aumento durante a adolescência devido a fatores biológicos ${ }^{(13)}$.

De forma geral, existem dois mecanismos biológicos que, em consonância com os estímulos ambientais, são responsáveis pela regulação do sono: o circadiano (processo C) e o homeostático (processo S). De acordo com Carskadon et al ${ }^{(12)}$, o processo $\mathrm{S}$ está relacionado a um aumento da propensão ao sono durante o dia e sofre o efeito de possíveis débitos de sono. Já o processo $\mathrm{C}$, regulado pelo sistema de temporização circadiano, é responsável pelo aumento da propensão do sono na fase escura do dia. $\mathrm{Na}$ adolescência, identificou-se ${ }^{(12)}$ uma maior lentidão na inibição da secreção de melatonina no início da fase clara do dia, especialmente nas fases tardias da puberdade, bem como um acúmulo mais lento da propensão para o sono durante o dia, o que pode levar a um atraso de fase.

Em síntese, as mudanças biológicas e comportamentais na adolescência levam a um atraso de fase que, considerando o contexto social e escolar, refletirá em uma diminuição das horas de sono e aumento da sonolência diurna excessiva, conforme ilustra a Figura $1^{(9,14,15)}$.

Apesar de substancial aprofundamento das questões envolvidas com o atraso de fase na adolescência e aumento da sonolência diurna excessiva, os inúmeros fatores envolvidos nesse fenômeno parecem não estar apresentados com clareza pela literatura, já que inúmeras são as variáveis que podem influenciar no padrão do ciclo vigília-sono e na sonolência diurna excessiva dos adolescentes. A clarificação desses fatores é ponto-chave para elaborar práticas educativas, intervenções no âmbito escolar e políticas públicas para a saúde dos adolescentes. Dessa forma, o objetivo desta pesquisa foi realizar um levantamento de estudos e apresentar, por meio de uma revisão sistemática, a prevalências e os fatores associados à sonolência diurna excessiva em adolescentes.

\section{Procedimentos}

A revisão sistemática de literatura foi realizada a partir das bases de dados PubMed e SciELO, sem recorte de período, utilizando os descritores "sonolência diurna excessiva" e "adolescentes". A pesquisa foi efetuada sem restrições de período e idioma. Além disso, a busca foi ampliada por pesquisa de estudos relevantes nas referências bibliográficas

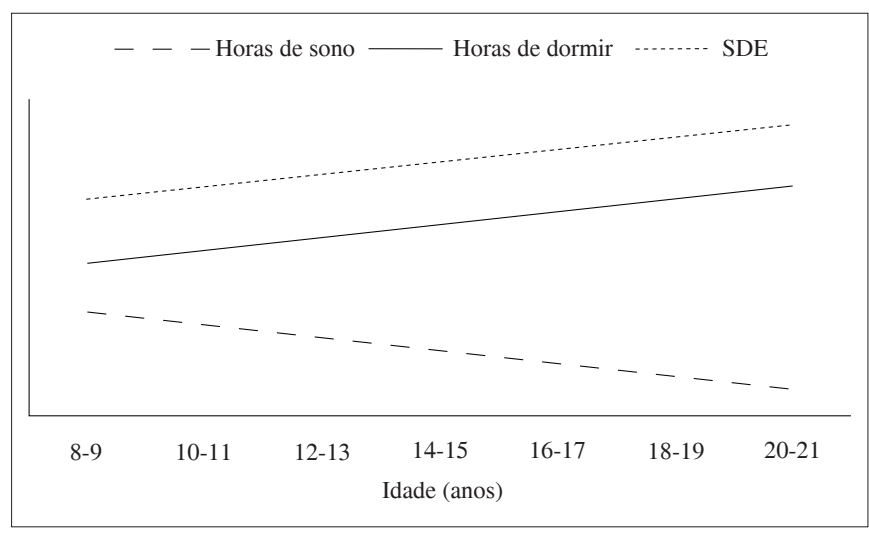

Figura 1 - llustração da tendência do atraso de fase, diminuição das horas de sono e aumento da SDE na adolescência. Baseado nos dados apresentados por Carskadon ${ }^{(9)}$, Andrade et $a^{\left({ }^{(14)}\right.}$, e Thorleifsdottir et $a /^{(15)}$, dentre outras pesquisas. 
dos artigos encontrados na busca inicial. Na primeira busca foram listados 394 artigos.

A partir dessa primeira etapa, os artigos selecionados para análise preencheram os seguintes critérios: apresentar dados originais, incluir adolescentes em suas amostras, mostrar resultados de prevalências ou incidência e/ou fatores associados à sonolência diurna excessiva, não serem voltados à investigação de populações com patologias específicas e possuírem, preferencialmente, amostras populacionais representativas. Outros estudos que não atenderam aos critérios de inclusão foram incluídos, de acordo com o julgamento dos autores. $\mathrm{O}$ total de artigos selecionados que atenderam a essas prerrogativas foi de 23 .

Verificou-se que a maioria dos artigos selecionados, utilizando-se as palavras-chave indicadas, não tratava exatamente do tema e sim de outros aspectos de sono, apontando a sonolência diurna excessiva como um possível desfecho para distúrbios do sono. Além disso, grande parte dos estudos investigou a sonolência diurna excessiva em grupos específicos com determinadas patologias, utilizando-a como uma variável de controle.

\section{Estudos sobre sonolência diurna excessiva}

Dos 23 estudos listados, aproximadamente $70 \%$ foram realizados com amostras orientais ou europeias. Com amostras brasileiras foram selecionadas duas investigações que analisaram pré-vestibulandos de Campo Grande (MS) ${ }^{(16)}$ e escolares de nove a 14 anos do município de Uruguaiana $(\mathrm{RS})^{(17)}$.

Com exceção dos estudos de Saarenpää-Heikkilä et a ${ }^{(18)} \mathrm{e}$ de Thorleifsdottir et $a l^{(15)}$, todos os trabalhos apresentaram desenho transversal. Na Tabela 1 encontra-se a síntese dos principais estudos analisados ${ }^{(3,5,7,15-28)}$.

As escalas de sonolência Epworth Sleepiness Scale e a School Sleep Habits Survey foram as mais utilizadas para análise da sonolência diurna excessiva. Alguns estudos populacionais, por exemplo, o de Gaina et al ${ }^{(24)}$, utilizaram questionários próprios, investigando a sonolência diurna excessiva com a seguinte questão: durante os dias em que você tem aula, quantas vezes sente muita sonolência (sono) na sala de aula? $\mathrm{O}$ adolescente poderia marcar uma das opções: sempre, frequentemente, às vezes ou nunca.

No estudo de Gaina et $a l^{(24)}$, a prevalência de sonolência diurna excessiva encontrada foi de $25,2 \%$ para a opção "sempre" e 47,6\% para a opção "frequentemente" da esca- la. Já a Pediatric Daytime Sleepiness Scale foi utilizada como medida contínua no estudo de Perez-Chada $e t$ al ${ }^{(4)}$, não classificando os sujeitos em sonolentos e não-sonolentos, comparando-se, na análise, os quartis dos escores. Vale ressaltar que o uso de diferentes instrumentos de diagnóstico pode comprometer a comparação entre as diversas realidades investigadas.

Assim, a prevalência de sonolência diurna excessiva encontrada nos estudos analisados apresentou grande variação. No estudo de Petry $e t a l^{(17)}$, identificou-se prevalência de $7,8 \%$, enquanto no de Souza et $a^{(16)}$ a prevalência foi de $55,8 \%$. Pode-se atribuir tal variação aos instrumentos utilizados, já que na pesquisa de Petry et al ${ }^{(17)}$ foi utilizado um questionário próprio, com apenas uma questão para avaliação da sonolência diurna excessiva, enquanto no de Souza $e t a l^{(16)}$ foi utilizada a Epworth Sleepiness Scale. Apesar disso, avaliando-se os demais estudos, não se verificou uma tendência de superestimativa ou subestimativa para algum instrumento específico. Como se tratam de dois estudos com amostras brasileiras, torna-se importante que outras pesquisas com casuística semelhante sejam realizadas para que se tenham parâmetros nacionais sobre o tema.

Os problemas relacionados ao sono mostraram-se fortemente associados à sonolência diurna excessiva, sendo que os mais investigados foram o ranger de dentes, a apneia, os roncos, os pesadelos e a insônia. Johnson e Roth ${ }^{(5)}$ verificaram, em amostra de 1.014 adolescentes de Michigan (Estados Unidos), que jovens com mais sonolência (quarto quartil de uma escala contínua) possuíam mais chance $(\mathrm{OR}=3,07$; IC95\% $=1,72-5,46)$ de apresentarem desordens durante o sono, mesmo com correções por sexo, idade, gênero, etnia e renda familiar.

Os estudos apresentaram outros fatores associados à sonolência diurna excessiva como, por exemplo, o gênero do adolescente e altos valores de IMC. É importante salientar que a maior parte dos estudos não analisou a sonolência diurna excessiva como variável "desfecho" e a maioria das informações sobre essa variável provém de estudos que utilizam os padrões de sono como variável dependente. Nesse contexto, muitos autores não mostram associações de todas as variáveis investigadas com a sonolência diurna excessiva.

Em relação ao gênero, as maiores prevalências foram encontradas nas meninas, como no estudo de Chung e Cheung ${ }^{(26)}$. Altos valores de índice de massa corporal (IMC) foram apontados como importante variável no estudo de Beebe et $a^{(29)}$. A forma de deslocamento até a escola foi analisada apenas pelo estudo de Chung e Cheung ${ }^{(26)}$ e, apesar 
disso, os pontos de corte para análise dessa variável não foram apresentados no artigo.

Poucas investigações com amostras brasileiras foram listadas. $\mathrm{O}$ estudo de Teixeira $e t a^{(8)}$, embora não tenha sido selecionado para avaliação na revisão sistemática e não conter uma amostra representativa, identificou altas prevalências de sonolência em adolescentes de São Paulo, trabalhadores e nãotrabalhadores, chegando a mais de $70 \%$ em alguns dias da

Tabela 1 - Síntese dos principais estudos selecionados sobre sonolência diurna excessiva (SDE)

\begin{tabular}{|c|c|c|c|c|}
\hline $\begin{array}{l}\text { Autores/ano de } \\
\text { publicação }\end{array}$ & $\begin{array}{l}\text { Amostral } \\
\text { nacionalidade }\end{array}$ & Instrumento* & $\begin{array}{c}\text { Prevalências } \\
(\%)\end{array}$ & Fatores associados \\
\hline Saarenpää-Heikkilä et a/(18) & 214, Finlândia & Questionário & n.a. & Problemas com sono \\
\hline Mantz et a/(19) & 386, França & Questionário & 38,0 & n.a. \\
\hline Giannotti et $a^{(20)}$ & 1.747, Itália & $\begin{array}{l}\text { School Sleep } \\
\text { Habits Survey }\end{array}$ & n.a. & $\begin{array}{l}\text { Cronotipo; distúrbios } \\
\text { psiquiátricos menores }\end{array}$ \\
\hline Thorleifsdottir et a/ ${ }^{(15)}$ & 668, Islândia & Questionário & n.a. & $\begin{array}{l}\text { Cochilos; horas de sono; } \\
\text { assistir TV }\end{array}$ \\
\hline Shin et $a^{(21)} ;$ Joo et $a^{(2)}$ & 3.871, Coreia do Sul & $\begin{array}{l}\text { Epworth } \\
\text { Sleepiness Scale }\end{array}$ & 15,9 & $\begin{array}{l}\text { Problemas com o sono; } \\
\text { baixo rendimento escolar }\end{array}$ \\
\hline Gau et a/(22) & 1.572, Taiwan & Questionário & n.a. & Horário de dormir \\
\hline Yang et $a^{(23)}$ & 1.457, Coreia & $\begin{array}{l}\text { School Sleep } \\
\text { Habits Survey }\end{array}$ & 17,1 & Sexo \\
\hline Gaina et a/(6) & 9.718, Japão & Questionário & n.a. & $\begin{array}{l}\text { Latência de sono maior que } \\
20 \text { minutos }\end{array}$ \\
\hline Gibson et al(7) & 3.235, Canadá & $\begin{array}{l}\text { Epworth } \\
\text { Sleepiness Scale }\end{array}$ & 41,5 & $\begin{array}{l}\text { Sexo; horas de sono; } \\
\text { rotina na hora de dormir; } \\
\text { ficar estudando até tarde; } \\
\text { consumo de cafeína }\end{array}$ \\
\hline Johnson e Roth ${ }^{(5)}$ & 1.014, EUA & Questionário & n.a. & Problemas com o sono \\
\hline Souza et a/(16) & 378, Brasil & $\begin{array}{l}\text { Epworth } \\
\text { Sleepiness Scale }\end{array}$ & 55,8 & Tabagismo; etilismo \\
\hline Perez-Chada et a/(4) & 2.884, Argentina & $\begin{array}{l}\text { Pediatric } \\
\text { Daytime } \\
\text { Sleepiness Scale }\end{array}$ & n.a. & $\begin{array}{l}\text { Problemas com o sono, } \\
\text { IMC e baixo desempenho } \\
\text { escolar, poucas horas de } \\
\text { sono }\end{array}$ \\
\hline Russo et a/(25) & 1.073, Itália & $\begin{array}{l}\text { School Sleep } \\
\text { Habits Survey }\end{array}$ & n.a. & $\begin{array}{l}\text { Problemas com o sono; } \\
\text { cronotipo }\end{array}$ \\
\hline Gaina et $a^{(24)}$ & 9.261, Japão & Questionário & 25,2 & $\begin{array}{l}\text { Problemas com o sono; } \\
\text { sexo; IMC; hábitos } \\
\text { alimentares; problemas com } \\
\text { o sono; nível de atividade } \\
\text { física e horas frente à TV }\end{array}$ \\
\hline Chung e Cheung ${ }^{(26)}$ & 1.629, China & $\begin{array}{l}\text { Epworth } \\
\text { Sleepiness Scale }\end{array}$ & 41,9 & $\begin{array}{l}\text { Cronotipo; sexo, idade, } \\
\text { estresse, deslocamento para } \\
\text { a escola, trabalho }\end{array}$ \\
\hline Gupta et $a /^{(27)}$ & 1.920, Índia & Questionário & 54,2 & Idade; série escolar \\
\hline Liu et $a /^{(3)}$ & 1.056, China & $\begin{array}{l}\text { School Sleep } \\
\text { Habits Survey }\end{array}$ & 17,9 & $\begin{array}{l}\text { Problemas com o sono; } \\
\text { série escolar }\end{array}$ \\
\hline Petry et a/(17) & 998, Brasil & Questionário & 7,8 & $\begin{array}{l}\text { Problemas com o sono; } \\
\text { respiração oral diurna; baixo } \\
\text { desempenho escolar }\end{array}$ \\
\hline Loessl et $a^{(28)}$ & 601, Alemanha & $\begin{array}{l}\text { School Sleep } \\
\text { Habits Survey }\end{array}$ & n.a. & $\begin{array}{l}\text { Menos de nove horas de } \\
\text { sono }\end{array}$ \\
\hline
\end{tabular}

n.a.: não apresentado. *questionário: instrumento próprio para os estudos que, em geral, avalia a SDE por meio da pergunta: durante os dias escolares, quantas vezes você sente muita sonolência? Quase sempre (ou sempre); frequentemente; raramente; nunca. 
semana. Além das maiores prevalências de sonolência terem sido verificadas nos adolescentes trabalhadores, os fatores associados foram o gênero, o consumo de álcool, dificuldades para adormecer e ir dormir após a meia-noite.

Outro estudo com sonolência diurna excessiva em amostra brasileira, já referido anteriormente, foi publicado por Souza et $a l^{(30)}$ e avaliou 408 sujeitos no município de Campo Grande. No entanto, apenas 25 sujeitos da amostra eram adolescentes, sendo o restante de adultos. Nesse estudo, a prevalência de sonolência diurna excessiva foi de 18,9\% e não houve associação significativa com o uso de hipnóticos, insônia, IMC, gênero, idade, escolaridade, classe socioeconômica, estado civil, ocupação e uso de meios alternativos para dormir melhor. Houve somente associação entre sonolência diurna excessiva e insônia para o sexo masculino. Já no estudo de Petry et al ${ }^{(23)}$, a prevalência encontrada foi de $7,8 \%$, sendo os problemas respiratórios e o baixo desempenho escolar os principais fatores associados. De forma geral, os estudos com amostras brasileiras não são conclusivos quanto às prevalências e aos principais fatores associados à sonolência diurna excessiva.

Apenas dois estudos com medidas de incidência foram encontrados. $\mathrm{Na}$ pesquisa de Saarenpää-Heikkilä et $a l^{(18)}$, um alto desempenho escolar foi identificado como fator de risco para o surgimento de novos casos de sonolência diurna excessiva, considerando um intervalo de dois anos de análise. Embora a metodologia do estudo tenha sido limitada, já que não foram utilizadas análises multivariadas ajustadas e a amostra foi de conveniência, os autores especulam que o estresse dos trabalhos escolares relacionados a um alto desempenho acadêmico pode desencadear a sonolência diurna excessiva em crianças e adolescentes que não a apresentavam anteriormente.

O estudo de acompanhamento com análise mais aprofundada encontrado foi o de Thorleifsdottir et al ${ }^{(15)}$, com coorte de acompanhamento de cinco e dez anos. Nessa pesquisa, verificou-se ser a idade uma variável com grande poder preditivo das variações de tempo total de sono e horário de dormir nos adolescentes, especialmente, a partir dos nove anos de idade. Os fatores associados à sonolência diurna excessiva, além da idade, foram as horas de sono e, em menor grau, o número de horas frente à televisão, embora o estudo tenha se fixado quase exclusivamente aos hábitos de sono, controlando poucas variáveis de confusão.

Um aumento da sonolência de acordo com a idade, durante a adolescência, foi identificado em grande parte dos estudos e parece estar bem definido. Essa tendência de aumento da sonolência diurna excessiva com o passar da puberdade pode ter levado Grupta et a $l^{(27)}$ e Liu et $a l^{(3)}$ a identificarem maior prevalência de sonolência diurna excessiva em séries escolares mais avançadas, o que leva à necessidade de correções estatísticas em futuros estudos com essas variáveis.

Com exceção do estudo de Gibson $e t$ al ${ }^{(7)}$, a preocupação com o nível de sonolência em diferentes períodos do dia não foi explicitada. Gibson et a $l^{(7)}$ identificaram que a prevalência geral de sonolência diurna excessiva de $41,5 \%$, chegando a $68 \%$ quando avaliada nas primeiras horas da manhã.

A análise dos estudos selecionados não permitiu identificar um ponto de corte padrão para o número de horas de sono ideal como medida protetora de sonolência diurna excessiva. No estudo de Loessl et a ${ }^{(28)}$ menos de nove horas de sono foi um fator associado a altos níveis de sonolência diurna excessiva. Já para Joo et al ${ }^{(2)}$, um dos principais fatores associados foi a insuficiência de sono percebida, mas o tempo total de sono foi semelhante nos jovens com e sem sonolência diurna excessiva, o que indica uma ausência de proteção desse fator. Apesar disso, os estudos analisados mostraram uma tendência preocupante de diminuição das horas de sono, como na análise de jovens argentinos ${ }^{(4)}$, na qual aproximadamente $50 \%$ deles dormiam menos de oito horas durante a semana.

Conforme Wolfson et $a l^{(10)}$, com o início da adolescência, muitos jovens necessitam mais de nove horas de sono e os horários escolares podem comprometer de forma importante o total de horas dormidas. Wolfson et al ${ }^{(10)}$ investigaram o padrão do ciclo vigília-sono de 250 jovens cujo horário de início das aulas era $7 \mathrm{~h} 15$ versus $8 \mathrm{~h} 37$ da manhã. Embora os alunos apresentassem horários de dormir e hábitos gerais de sono semelhantes, o grupo que iniciava suas atividades escolares mais tarde apresentava um período de sono maior e menos sonolência diurna excessiva.

Limitações nas seleções dos adolescentes nos estudos analisados foram verificadas, sendo que, na maioria, a seleção amostral se deu por conveniência. Além disso, poucos pesquisadores tiveram a preocupação de realizar análises ajustadas para identificar fatores realmente importantes para a sonolência diurna excessiva e diminuir a influência de variáveis de confusão. A totalidade dos estudos que apresentou análises de regressão utilizou razão de chances (Odds Ratio) obtida por meio de regressão logística, o que pode superestimar os resultados quando as razões forem maiores que 1 e subestimálas quando menores que 1 , especialmente em estudos com prevalências superiores a $10 \%$, conforme demonstrado por Barros e Hirakata ${ }^{(31)}$ e discutido em editorial da American 
Journal of Epidemiology $y^{(32)}$. Como as prevalências de sonolência diurna excessiva normalmente são altas, o cálculo de razões de prevalências por meio de regressão de Poisson pode ser uma opção mais coerente de análise.

\section{Considerações finais}

Uma carência de dados de prevalências, incidências e fatores associados e de risco para a sonolência diurna excessiva foi identificada, especialmente em populações brasileiras. Os fatores associados apresentados, com exceção dos problemas de sono, não indicaram com clareza possibilidades de intervenção para minimizar os efeitos negativos da sonolência diurna excessiva em adolescentes. Estudos epidemiológicos

\section{Referências bibliográficas}

1. D'Alessandro R, Rinaldi R, Cristina E, Gamberini G, Lugaresi E. Prevalence of excessive daytime sleepiness an open epidemiological problem. Sleep 1995;18:389-91.

2. Joo S, Shin C, Kim J, Yi H, Ahn Y, Park M et al. Prevalence and correlates of excessive daytime sleepiness in high school students in Korea. Psychiatry Clin Neurosci 2005;59:433-40.

3. Liu X, Zhao Z, Jia C, Buysse DJ. Sleep patterns and problems among Chinese adolescents. Pediatrics 2008;121:1165-73.

4. Perez-Chada D, Perez-Lloret S, Videla AJ, Cardinali D, Bergna MA, FernándezAcquier $\mathrm{M}$ et al. Sleep disordered breathing and daytime sleepiness are associated with poor academic performance in teenagers. A study using the Pediatric Daytime Sleepiness Scale (PDSS). Sleep 2007;30:1698-703.

5. Johnson EO, Roth T. An epidemiologic study of sleep-disordered breathing symptoms among adolescents. Sleep 2006;29:1135-42.

6. Gaina A, Sekine M, Kanayama H, Takashi Y, Hu L, Sengoku K et al. Morningevening preference: sleep pattern spectrum and lifestyle habits among japanese junior high school pupils. Chronobiol Int 2006;23:607-21.

7. Gibson ES, Powles AC, Thabane L, O'Brien S, Molnar DS, Trajanovic N et al. "Sleepiness" is serious in adolescence: two surveys of 3235 Canadian students. BMC Public Health 2006;6:116.

8. Teixeira LR, Lowden A, Lemos TS, Nagai R, Moreno CR, Latorre MR et al. Sleep and sleepiness among working and non-working high school evening students. Chronobiol Int 2007;24:99-113.

9. Carskadon MA, Vieira C, Acebo C. Association between puberty and delayed phase preference. Sleep 1993;16:258-62.

10. Wolfson AR, Spaulding NL, Dandrow C, Baroni EM. Middle school start times: the importance of a good night's sleep for young adolescents. Behav Sleep Med 2007;5:194-209.

11. Moore M, Meltzer LJ. The sleepy adolescent: causes and consequences of sleepiness in teens. Paediatr Respir Rev 2008;9:114-20.

12. Carskadon MA, Acebo C, Jenni OC. Regulation of adolescent sleep: implications for behavior. Ann N Y Acad Sci 2004;1021:276-91.

13. Campbell IG, Higgins LM, Trinidad JM, Richardson P, Feinberg I. The increase in longitudinally measured sleepiness across adolescence is related to the maturational decline in low-frequency EEG power. Sleep 2007;30:1677-87.

14. Andrade MM, Benedito-Silva AA, Domenice S, Arnhold IJ, Menna-Barreto L. Sleep characteristics of adolescents: a longitudinal study. J Adolesc Health 1993;14:401-6.

15. Thorleifsdottir B, Björnsson JK, Benediktsdottir B, Gislason T, Kristbjarnarson H. Sleep and sleep habits from childhood to young adulthood over a 10-year period. J Psychosom Res 2002;53:529-37. com desenhos amostrais e tratamentos estatísticos adequados são necessários, bem como uma padronização dos instrumentos para mensurar o problema.

Variáveis como as características do deslocamento até a escola, os hábitos alimentares no café da manhã, o percentual de adiposidade corporal, o histórico dos jovens em relação aos seus horários escolares nos anos anteriores, o ambiente onde o jovem dorme, a localização (urbana/rural) da escola, o cronotipo dos pais, os transtornos psiquiátricos menores, o tabagismo e etilismo, a inserção no mundo do trabalho, dentre outras, que podem interferir no padrão do ciclo vigília-sono e na sonolência diurna excessiva, foram pouco estudados ou ainda não foram analisadas, especialmente em estudos de abrangência populacional.

16. Souza JC, Souza N, Arashiro ESH, Schaedler R. Excessive daytime sleepiness in senior high school students. J Bras Psiquiatr 2007;56:184-7.

17. Petry C, Pereira MU, Pitrez PM, Jones MH, Stein RT. The prevalence of symptoms of sleep-disordered breathing in Brazilian schoolchildren. J Pediatr (Rio J) 2008;84:123-9.

18. Saarenpää-Heikkilä O, Laippala $P$, Koivikko M. Subjective daytime sleepiness in schoolchildren. Fam Pract 2000;17:129-33.

19. Mantz J, Muzet A, Winter AS. The characteristics of sleep-wake rhythm in adolescents aged 15-20 years. A survey made at school during ten consecutive days. Arch Pediatr 2000;7:256-62.

20. Giannotti F, Cortesi F, Sebastiani T, Ottaviano S. Circadian preference, sleep and daytime behaviour in adolescence. J Sleep Res 2002;11:191-9.

21. Shin C, Kim J, Lee S, Ahn Y, Joo S. Sleep habits, excessive daytime sleepiness and school performance in high school students. Psychiatry Clin Neurosci 2003;57:451-3.

22. Gau SS, Soong WT, Merikangas KR. Correlates of sleep-wake patterns among children and young adolescents in Taiwan. Sleep 2004;27:512-9.

23. Yang CK, Kim JK, Patel SR, Lee JH. Age-related changes in sleep/wake patterns among Korean teenagers. Pediatrics 2005;115 (Suppl 1):250-6.

24. Gaina A, Sekine M, Hamanishi S, Chen X, Wang H, Yamagami T, Kagamimori S. Daytime sleepiness and associated factors in Japanese school children. J Pediatr 2007;151:518-22.

25. Russo PM, Bruni O, Lucidi F, Ferri R, Violani C. Sleep habits and circadian preference in Italian children and adolescents. J Sleep Res 2007;16:163-9.

26. Chung KF, Cheung MM. Sleep-wake patterns and sleep disturbance among Hong Kong Chinese adolescents. Sleep 2008;31:185-94.

27. Gupta R, Bhatia MS, Chhabra V, Sharma S, Dahiya D, Semalti K et al. Sleep patterns of urban school-going adolescents. Indian Pediatr 2008;45:183-9.

28. Loessl B, Valerius G, Kopasz M, Hornyak M, Riemann D, Voderholzer U. Are adolescents chronically sleep-deprived? An investigation of sleep habits of adolescents in the Southwest of Germany. Child Care Health Dev 2008;34:549-56.

29. Beebe DW, Lewin D, Zeller M, McCabe M, MacLeod K, Daniels SR et al. Sleep in overweight adolescents: shorter sleep, poorer sleep quality, sleepiness, and sleep-disordered breathing. J Pediatr Psychol 2007;32:69-79.

30. Souza JC, Magna LA, Reimão R. Excessive daytime sleepiness in Campo Grande general population, Brazil. Arq Neuropsiquiatr 2002;60:558-62.

31. Barros AJ, Hirakata VN. Alternatives for logistic regression in cross-sectional studies: an empirical comparison of models that directly estimate the prevalence ratio. BMC Med Res Methodol 2003;3:21.

32. Spiegelman D, Hertzmark E. Easy SAS calculations for risk or prevalence ratios and differences. Am J Epidemiol 2005;162:199-200. 